\title{
Remark on the additivity conjecture for the quantum depolarizing channel
}

\author{
G.G. Amosov
}

November 13, 2018

\begin{abstract}
We consider bistochastic quantum channels generated by unitary representations of the discret group. The proof of the additivity conjecture for the quantum depolarizing channel $\Phi$ based on the decreasing property of the relative entropy is given. We show that the additivity conjecture holds for the channel $\Xi=\Psi \circ \Phi$, where $\Psi$ is the phase damping.
\end{abstract}

\section{Introduction.}

Let $H, \operatorname{dim} H=l<+\infty$, be a complex Hilbert space. Denote $\sigma(H), \operatorname{Proj}(H)$ and $I_{H}$ the set of all states, i.e. positive unite-trace operators, the set of all one-dimensional projections and the identity operator in $H$, respectively. By a quantum channel $\Psi$ in $H$ we mean a linear map on $\sigma(H)$ such that the conjugate linear map $\Psi^{*}$ defined on the algebra of all bounded operators $B(H)$ on $H$ is completely positive and unital (preserving the identity operator). If $\Psi\left(\frac{1}{l} I_{H}\right)=\frac{1}{l} I_{H}$, then the channel $\Psi$ is said to be bistochastic. The entropy upper bound for the channel $\Psi$ is defined by the formula

$$
C_{1}(\Psi)=\sup _{x_{j} \in \sigma(H), \pi} S\left(\sum_{j=1}^{r} \pi_{j} \Psi\left(x_{j}\right)\right)-\sum_{j=1}^{r} \pi_{j} S\left(\Psi\left(x_{j}\right)\right),
$$

where $S(x)=-\operatorname{Tr} x \log x$ is the von Neumann entropy of $x$ and the supremum is taken over all probability distributions $\pi=\left(\pi_{j}\right)_{j=1}^{r}, 0 \leq$ 
$\pi_{j} \leq 1, \sum_{j=1}^{r} \pi_{j}=1$. The additivity conjecture states that for any two channels $\Phi$ and $\Psi$

$$
C_{1}(\Phi \otimes \Psi)=C_{1}(\Phi)+C_{1}(\Psi) .
$$

If the additivity conjecture holds, one can easily find the capacity $C(\Psi)$ of the channel $\Psi$ by the formula $C(\Psi)=\lim _{n \rightarrow+\infty} \frac{C_{1}\left(\Psi^{\otimes n}\right)}{n}=C_{1}(\Psi)$ (see [1]). In [1] the additivity conjecture is proved for so called c-q and q-c channels. It follows from the definition of $C_{1}$ that

$$
C_{1}(\Psi) \leq S\left(\frac{1}{l} I_{H}\right)-\inf _{x \in \sigma(H)} S(\Psi(x)) .
$$

The equality at the last formula was stated in [2] for bistochastic qubit channels, in [4] for so-called qudit channels, in [5] for covariant channels. Moreover it was proved in [6] that for an arbitrary channel the additivity conjecture for the quantity $C_{1}$ is equivalent to the additivity conjecture for the entropy infimum

$$
\inf _{x \in \sigma\left(H_{1} \otimes H_{2}\right)} S(\Phi \otimes \Psi(x))=\inf _{x_{1} \in \sigma\left(H_{1}\right)} S\left(\Phi\left(x_{1}\right)\right)+\inf _{x_{2} \in \sigma\left(H_{2}\right)} S\left(\Psi\left(x_{2}\right)\right) .
$$

Fix a number $p>1$. Then one can define $l_{p}$-norm of the channel $\Psi$ by the formula $\|\Psi\|_{p}=\left(\sup _{x \in \operatorname{Proj}(H)} \operatorname{Tr} \Psi(x)^{p}\right)^{\frac{1}{p}}$. It is shown in [2] that the additivity conjecture is closely connected with the following multiplicativity conjecture which states for two channels $\Phi$ and $\Psi$

$$
\|\Phi \otimes \Psi\|_{p}=\|\Phi\|_{p}\|\Psi\|_{p} .
$$

In particular, if the multiplicativity conjecture holds for $p$ close to 1 , the additivity conjecture is true also. The multiplicativity conjecture for the depolarizing channel is proved in [3] for integer numbers of the index $p$. The additivity and multiplicativity conjectures are shown to be true for unital qubit channels in [8], for the quantum depolarizing channel in [9] and for the entanglement-breaking quantum channels in [10]. In [7] it is considered the connection of the additivity conjecture for arbitrary channels and for constrained channels. Nevertheless, it was no way allowing to check the conjectures for an arbitrary channel. In [11] it is given a counterexample to the multiplicativity conjecture. So one can expect that the additivity conjecture can be not true for some channels. 
We investigate bistochastic quantum channels generated by unitary representation of the discret group. For two states $\rho_{1}$ and $\rho_{2}$ let $S\left(\rho_{1}, \rho_{2}\right)=\operatorname{Tr} \rho_{1} \log \rho_{1}-\operatorname{Tr} \rho_{1} \log \rho_{2}$ be a relative entropy. The decreasing property of the relative entropy $S\left(\rho_{1}, \rho_{2}\right)$ states (see [12])

$$
S\left(\Xi\left(\rho_{1}\right), \Xi\left(\rho_{2}\right)\right) \leq S\left(\rho_{1}, \rho_{2}\right)
$$

for a quantum channel $\Xi$ and any two states $\rho_{1}$ and $\rho_{2}$. In particular, if the channel $\Xi$ is bistochastic, it means that

$$
S(\Xi(\rho)) \geq S(\rho)
$$

for an arbitrary state $\rho$. The quantum depolarizing channel $\Phi$ is a unique channel satisfying the covariance property $U \Phi(x) U^{*}=$ $\Phi\left(U x U^{*}\right)$ for any unitary operator $U$. We prove the additivity conjecture for $\Phi$ by means of the decreasing property of the relative entropy. We show that the additivity property of the entropy upper bound for the depolarizing channel $\Phi$ allows to prove the additivity conjecture for the channels $\Xi=\Psi \circ \Phi$, where $\Psi$ is the phase damping.

\section{The main result.}

The quantum depolarizing channel is defined by the formula $\Phi(x)=$ $(1-p) x+\frac{p}{l} I_{H}, x \in \sigma(H), 0<p \leq \frac{l^{2}}{l^{2}-1}$. Fix the basis $e_{j}, 1 \leq j \leq l$, in $H$ and define a linear map $\Psi$ by the formula

$$
\begin{gathered}
\Psi\left(\left|e_{s}><e_{j}\right|\right)=q_{|s-j|}\left|e_{s}><e_{j}\right|, 1 \leq s, j \leq l,|s-j|<l-1, \\
\Psi\left(\left|e_{1}><e_{l}\right|\right)=q_{1}\left|e_{1}><e_{l}\right|, \Psi\left(\left|e_{l}><e_{1}\right|\right)=q_{1}\left|e_{l}><e_{1}\right|, \\
q_{0}=1,0 \leq q_{j} \leq 1,1 \leq j \leq l-1 .
\end{gathered}
$$

If $\Psi$ is completely positive, then we shall call its restriction to $\sigma(H)$ by a phase damping. The phase damping is a bistochastic channel.

In [9] it is proved that the additivity and multiplicativity conjecture hold for the depolarizing channel. We shall give an alternative proof of this statement. Moreover, we shall prove the following theorem:

Theorem. The additivity conjecture holds for any channel of the form $\Xi=\Psi \circ \Phi$, where $\Phi$ is the quantum depolarizing channel and $\Psi$ is the phase damping. 
Denote $\Xi=\Xi\left(\lambda_{1}, \lambda_{2}, \lambda_{3}\right)$ the bistochastic qubit channel with the parameters $\left(\lambda_{1}, \lambda_{2}, \lambda_{3}\right)$ acting by the formula

$$
\Xi\left\{\left(\begin{array}{cc}
a & b+i c \\
b-i c & d
\end{array}\right)\right\}=\left(\begin{array}{cc}
\frac{1+\lambda_{3}}{2} a+\frac{1-\lambda_{3}}{2} d & \lambda_{1} b+i \lambda_{2} c \\
\lambda_{1} b-i \lambda_{2} c & \frac{1-\lambda_{3}}{2} a+\frac{1+\lambda_{3}}{2} d
\end{array}\right),
$$

where matrices written in some basis $e_{1}, e_{2}$ of the Hilbert space $H, \operatorname{dim} H=2, a, b, c, d \in \mathbb{R}$. Let us assume that $\lambda_{1}=\lambda_{2}$ and $\left|\lambda_{1}\right| \leq \lambda_{3}$. Then $\Xi$ can be represented as $\Xi\left(\lambda_{1}, \lambda_{1}, \lambda_{3}\right)=\Xi\left(\frac{\lambda_{1}}{\lambda_{3}}, \frac{\lambda_{1}}{\lambda_{3}}, 1\right) \circ$ $\Xi\left(\lambda_{3}, \lambda_{3}, \lambda_{3}\right)$. Notice that $\Xi\left(\lambda_{3}, \lambda_{3}, \lambda_{3}\right)$ is the depolarizing channel and $\Xi\left(\frac{\lambda_{1}}{\lambda_{3}}, \frac{\lambda_{1}}{\lambda_{3}}, 1\right)$ is the phase damping. It follows from Theorem that the additivity and multiplicativity conjectures hold for the qubit channels of the form defined above.

\section{Bistochastic quantum channels gen- erated by unitary representations of the discret group.}

Let $G$ and $G_{0} \subset G$ be a finite discret group and its normal subgroup. Denote by $e$ the unit of the group $G$. Suppose that $|G|=l^{2},\left|G_{0}\right|=l$, and there exists an irreducible projective unitary representation $g \rightarrow$ $U_{g}$ of the group $G$ in the Hilbert space $H$. Consider the factor-group $G / G_{0}$ consisting of equivalency classes $[g]=\left\{g g_{0} \mid g_{0} \in G_{0}\right\}$. It follows from the definition that $\left|G / G_{0}\right|=l$. Take one element in each class $[g]$ and enumerate these elements in an arbitrary order. Then we get the set $G_{1}=\left\{g_{1} \equiv e, g_{2}, \ldots, g_{l}\right\}$ such that the classes $\left[g_{s}\right]$ generate $G / G_{0}$. We shall suppose that $G_{1}$ is a subgroup of $G$. Notice that there exist $l$ ways to construct the group $G_{1}$ accordingly to a number of elements in each coset $[g]$.

Example. The discret Weyl group.

Put $G=\mathbb{Z}_{l} \oplus \mathbb{Z}_{l}$ and $G_{0}=\mathbb{Z}_{l} \oplus 0, G_{1}=0 \oplus \mathbb{Z}_{l}$. Notice that $G_{0}$ and $G_{1}$ are normal subgroups of $G$. On the other hand, every element $1 \oplus s$ generates a subgroup of $G$ for $0 \leq s \leq l-1$. We shall denote this subgroup by $G_{0 k}, 0 \leq k \leq l-1$, such that $G_{00} \equiv G_{0}$. Thus, $G_{0 k}=\{s \oplus s k, 0 \leq s \leq l-1\}$. Notice that if $0 \leq k \leq l-1$ and $s \neq 0$ the elements $s \oplus s k \in G_{0 k}$ run the coset $[s \oplus 0]$ of the factor-group $G / G_{1}$. Fix a basis $\left(e_{j}\right)_{j=1}^{l}$ in the Hilbert space $H$ and define unitary 
operators $U_{g}, g \in G$, as follows

$$
\begin{gathered}
U_{g} e_{k}=e_{(k+s) \bmod (l))}, g=s \oplus 0 \in G_{0}, \\
U_{g} e_{k}=e^{\frac{2 \pi i s k}{l}} e_{k}, g=0 \oplus s \in G_{1} .
\end{gathered}
$$

Then, the formula $U_{k \oplus s}=U_{k \oplus 0} U_{0 \oplus s}, k \oplus s \in G$, defines an irreducible projective unitary representation of the group $G$.

Let us define completely positive linear maps $E_{0}$ and $E_{1}$ as follows

$$
E_{k}(x)=\frac{1}{l} \sum_{g \in G_{k}}^{l} U_{g} x U_{g}^{*}, x \in \sigma(H), k=0,1 .
$$

The map $E_{k}$ is a conditional expectation to the algebra of fixed elements $\mathcal{A}_{k}=\left\{x \in B(H) \mid U_{g} x U_{g}^{*}=x, g \in G_{k}\right\}, k=0,1$. Because the representation $g \rightarrow U_{g}$ is irreducible, we get the equality

$$
\sum_{k=1}^{l} U_{g_{k}} E_{0}(x) U_{g_{k}}^{*}=I_{H}, x \in \sigma(H) .
$$

Hence, the operators $X_{k}=U_{g_{k}} E_{0}(x) U_{g_{k}}, 1 \leq k \leq l$, form a (non orthogonal, in general) resolution of the identity. Take a probability distribution $\mu=\left\{\mu_{g}, g \in G\right\}$ on the group $G$ and define a bistochastic channel $\Phi$ by the formula

$$
\Phi(x)=\sum_{g \in G} \mu_{g} U_{g} x U_{g}^{*}, x \in \sigma(H) .
$$

Let us define a probability distribution $\lambda=\left\{\lambda_{k}, 1 \leq k \leq l\right\}$ on the factor-group $G / G_{0}$ as follows

$$
\lambda_{k}=\sum_{g \in G_{0}} \mu_{g_{k} g_{0}}, 1 \leq k \leq l .
$$

Consider the channel $\Phi \otimes I d$ in the Hilbert space $\tilde{H}=H \otimes K$. Here and in the following we denote by $I d$ the ideal (identity) channel.

Proposition 1. Suppose that the numbers $\frac{\mu_{g_{k}}}{\lambda_{k}}=\epsilon_{g}, g \in G_{0}$, do not depend on a choice of $k, 1 \leq k \leq l$. Then, the following inequality holds,

$$
S((\Phi \otimes I d)(x)) \geq S\left(\sum_{k=1}^{l} \lambda_{k}\left(U_{g_{k}} \otimes I_{K}\right) x\left(U_{g_{k}}^{*} \otimes I_{K}\right)\right), x \in \sigma(H \otimes K) .
$$


Proof.

Let us define a bistochastic channel $\Psi$ by the formula

$$
\Psi(x)=\sum_{g \in G_{0}} \epsilon_{g} U_{g} x U_{g}^{*}, x \in \sigma(H) .
$$

Then,

$$
S(\Phi \otimes I d(x))=S\left((\Psi \otimes I d)\left(\sum_{k=1}^{n} \lambda_{k} U_{g_{k}} x U_{g_{k}}^{*}\right)\right) \geq S\left(\sum_{k=1}^{n} \lambda_{k} U_{g_{k}} x U_{g_{k}}^{*}\right)
$$

by means of the decreasing property of the relative entropy.

Now put $\Phi(x)=\sum_{k=1}^{l} \lambda_{k} U_{g_{k}} x U_{g_{k}}, x \in \sigma(H), A_{1}=\left\{x \mid U_{g_{k}} x U_{g_{k}}^{*}=\right.$ $x, 1 \leq k \leq l\}$ and $E_{1}$ is the conditional expectation (1) to $A_{1}$. Then, $\tilde{E}_{1}(x)=E_{1} \otimes I d(x)=\frac{1}{l} \sum_{g \in G_{0}}^{l}\left(U_{g} \otimes I_{K}\right) x\left(U_{g} \otimes I_{K}\right), x \in \sigma(H)$, is a conditional expectation to the algebra $A_{1} \otimes B(K)$. Because $A_{1}$ is the algebra of fixed elements for the action of the group $\left(U_{g_{k}}\right)_{k=1}^{l}$, we get

$$
E_{1} \circ \Phi(x)=\Phi \circ E_{1}(x)=E_{1}(x), x \in \sigma(H) .
$$

Pick up the orthogonal resolution of the identity $\left(P_{k}\right)_{k=1}^{l}$ generating $A_{1}$.

\section{Proposition 2.}

$$
\begin{gathered}
S((\Phi \otimes I d)(x)) \geq-\sum_{k=1}^{l} \lambda_{k} \log \lambda_{k} \\
-\sum_{k=1}^{l} \operatorname{Tr}_{H}\left(\left(P_{k} \otimes I_{K}\right) \tilde{E}_{1}(x)\right) \log \operatorname{Tr}_{H}\left(\left(P_{k} \otimes I_{K}\right) \tilde{E}_{1}(x)\right)-\log (l) .
\end{gathered}
$$

Proof.

Let us define a quantum channel $\Xi_{x}$ in the Hilbert space $H \otimes K$ by the formula

$$
\Xi_{x}(\rho)=\sum_{k=1}^{l} \operatorname{Tr}\left(\left(P_{k} \otimes I_{K}\right) \rho\right)\left(U_{g_{k}} \otimes I_{K}\right) x\left(U_{g_{k}}^{*} \otimes I_{K}\right), \rho \in \sigma(H \otimes K) .
$$


Put $\rho=\sum_{k=1}^{l} \lambda_{k} P_{k} \otimes Q, \bar{\rho}=\frac{1}{l} \sum_{k=1}^{l} P_{k} \otimes Q=\left(\frac{1}{l}\right) I_{H} \otimes Q$, where $Q \in$ $\operatorname{Proj}(K)$. Then, $\Xi_{x}(\rho)=(\Phi \otimes I d)(x)$ and the relative entropy

$$
\begin{gathered}
S\left((\Phi \otimes I d)(x), \tilde{E}_{1}(x)\right)=S\left(\Xi_{x}(\rho), \Xi_{x}(\bar{\rho})\right) \leq \\
S(\rho, \bar{\rho})=\operatorname{Tr}(\rho \log \rho)-\operatorname{Tr}(\rho \log \bar{\rho})=\sum_{k=1}^{l} \lambda_{k} \log \lambda_{k}+\log (l) .
\end{gathered}
$$

On the other hand,

$$
\begin{gathered}
S\left((\Phi \otimes I d)(x), \tilde{E}_{1}(x)\right)=\sum_{k=1}^{l} \operatorname{Tr}((\Phi \otimes I d)(x) \log (\Phi \otimes I d)(x))- \\
\operatorname{Tr}\left((\Phi \otimes I d)(x) \log \tilde{E}_{1}(x)\right)=-S((\Phi \otimes I d)(x)) \\
-\operatorname{Tr}\left(\tilde{E}_{1}((\Phi \otimes I d)(x)) \log \tilde{E}_{1}(x)\right)= \\
-S((\Phi \otimes I d)(x))-\operatorname{Tr} \tilde{E}_{1}(x) \log \tilde{E}_{1}(x) .
\end{gathered}
$$

Here we use the equality $\tilde{E}_{1}((\Phi \otimes I d)(x))=\tilde{E}_{1}(x)$ in virtue of (5). Notice that $\tilde{E}_{1}(x)=\sum_{k=1}^{l} P_{k} \otimes x_{k}$, where $x_{k} \in \sigma(K)$. Then, $P_{k} \otimes x_{k}=$ $\tilde{E}_{1}(x) P_{k} \otimes I_{K}$. It follows that

$$
x_{k}=\operatorname{Tr}_{H}\left(\left(P_{k} \otimes I_{K}\right) \tilde{E}_{1}(x)\right), 1 \leq k \leq l .
$$

In this way,

$$
\tilde{E}_{1}(x)=\sum_{k=1}^{l} P_{k} \otimes \operatorname{Tr}_{H}\left(\left(P_{k} \otimes I_{K}\right) \tilde{E}_{1}(x)\right), x \in \sigma(H \otimes K) .
$$

To complete the proof, it is sufficiently to substitute (8) to (7) and compare the result with (6).

\section{The quantum depolarizing channel.}

Consider the quantum depolarizing channel $\Phi(x)=(1-p) x+$ $\frac{p}{l} \operatorname{Tr}(x), x \in \sigma(H)$. Due to the property $\frac{1}{l} \sum_{g \in G} U_{g} x U_{g}^{*}=I_{H}$ the channel $\Phi$ can be represented as $\Phi(x)=\left(1-\frac{l^{2}-1}{l^{2}} p\right) x+\sum_{g \in G, g \neq e} \frac{p}{l^{2}} U_{g} x U_{g}$. 
Hence, it has the form (3) with $\mu_{e}=1-\frac{l^{2}-1}{l^{2}} p, \mu_{g}=\frac{p}{l^{2}}, g \neq e$. Let $G$ be the discret Weyl group. Any element $g \neq e$ of the group $G$ belongs to one and only one subgroup $G_{0 k}$ or $G_{1}$. On the other hand, two elements $g_{k} \in G_{0 k}$ and $g_{r} \in G_{0 r}$ are connected by certain element $g \in G_{1}$ such that $g_{r}=g_{k}+g$. Thus, the direct sum $\oplus_{0 \leq k \leq l-1} G_{0 k}$ consists of $l$ units and one by one non-unit elements of $G$ except elements of $G_{1}$. The direct sum $\oplus_{g \in G_{1}, g \neq e, 0 \leq k \leq l-1}\left\{g+G_{0 k}\right\}$ consists of $l$ th copies of non-unit elements of $G_{1}$ and $(l-1)$ th copies of non-unit elements of $\left\{G_{0 k}, 0 \leq k \leq l-1\right\}$. Put $c_{0}=\frac{1}{l} \frac{1-\frac{l^{2}-1}{l^{2} p}}{1-\frac{l-1}{l} p}, c_{1}=\frac{1}{l} \frac{\frac{p}{l^{2}}}{1-\frac{l-1}{l} p}$, $\lambda_{0}=1-\frac{l-1}{l} p, \lambda_{k}=\frac{p}{l}, 1 \leq k \leq l-1$. Then, the depolarizing channel $\Phi$ can be represented as

$$
\Phi(x)=c_{0} \sum_{k=0}^{l-1} \Phi_{k}(x)+c_{1} \sum_{k=0}^{l-1} \sum_{s=1}^{l-1} U_{0 \oplus s} \Phi_{k}(x) U_{0 \oplus s}^{*}
$$

where

$$
\Phi_{k}(x)=\sum_{s=0}^{l-1} \lambda_{s} U_{s \oplus s k} x U_{s \oplus s k}^{*}, \quad 0 \leq k \leq l-1 .
$$

Notice that the first sum in (9) includes the actions of the elements from the direct sum $\oplus_{0 \leq k \leq l-1} G_{0 k}$, while the second sum includes the actions of the elements from $\oplus_{g \in G_{1}, g \neq e, 0 \leq k \leq l-1}\left\{g+G_{0 k}\right\}$.

Proposition 3. Let $\Phi$ be the quantum depolarizing channel. Then, given a state $x \in \sigma(H \otimes K)$ there exists the projection $P \in \operatorname{Proj}(H)$ such that $\rho=l \operatorname{Tr}_{H}((P \otimes I d) x) \in \sigma(K)$ and

$S(\Phi \otimes I d(x)) \geq-\left(1-\frac{l-1}{l} p\right) \log \left(1-\frac{l-1}{l} p\right)-(l-1) \frac{p}{l} \log \frac{p}{l}+S(\rho)$.

Proof.

Pick up a unitary operator $W$ in the Hilbert space $H$ such that the projection $\left.y=\left(W \otimes I_{K}\right) x\left(W^{*} \otimes I_{K}\right)\right)$ has the form

$$
y=\frac{1}{l} \sum_{k=1}^{l} P_{k} \otimes x_{k}, x_{k} \in \sigma(K) .
$$

This property is equivalent to the condition $\operatorname{WTr}_{K}(x) W^{*}=\frac{1}{l} I_{H}$. Using the covariance of the depolarizing channel we obtain $(\Phi \otimes I d)(x)=$ 
$\left(W^{*} \otimes I_{K}\right)(\Phi \otimes I d)(y)\left(W \otimes I_{K}\right)$. The representation (9) gives us the estimate

$$
S((\Phi \otimes I d)(x))=S((\Phi \otimes I d)(y)) \geq \inf _{0 \leq k \leq l-1} S\left(\left(\Phi_{k} \otimes I d\right)(y)\right) .
$$

Applying Proposition 2 to the projection $y$ we get the inequality

$$
\begin{aligned}
& S\left(\left(\Phi_{k} \otimes I d\right)(y)\right) \geq-\left(1-\frac{l-1}{l} p\right) \log \left(1-\frac{l-1}{l} p\right)-(l-1) \frac{p}{l} \log \frac{p}{l} \\
& -\sum_{k=1}^{l} \operatorname{Tr}_{H}\left(\left(P_{k} \otimes I_{K}\right) \tilde{E}_{1}(y)\right) \log \operatorname{Tr}_{H}\left(\left(P_{k} \otimes I_{K}\right) \tilde{E}_{1}(y)\right)-\log (l) .
\end{aligned}
$$

The condition (10) results in the formula $\operatorname{Tr}_{H}\left(\left(P_{k} \otimes I_{K}\right) \tilde{E}_{1}(y)\right)=$ $\frac{1}{l} y_{k}, y_{k} \in \sigma(K)$. Choose $k_{0}$ such that $\inf _{1 \leq k \leq l} S\left(y_{k}\right)=S\left(y_{k_{0}}\right)$. Put $P=W^{*} P_{k_{0}} W$, then (11) implies that

$$
\begin{aligned}
S\left(\left(\Phi_{k} \otimes I_{K}\right)(x)\right) \geq- & \left(1-\frac{l-1}{l}\right) \log \left(1-\frac{l-1}{l} p\right)-(l-1) \frac{p}{l} \log \frac{p}{l} \\
+ & S\left(l \operatorname{Tr}_{H}\left(P \otimes I_{K}\right) x\right) .
\end{aligned}
$$

The result follows.

Proposition 4. Let the following inequalities hold,

$$
q_{j} \leq \frac{1}{l-1}\left(1+\sum_{j=1}^{l-2} q_{j}\right) \equiv q, 1 \leq j \leq l-2,
$$

then the map $\Psi$ defined by means of (1) is completely positive.

Remark. If $q_{j}=Q=$ const, $1 \leq j \leq l-2$, then $q=\frac{1}{l-1}(1+(l-$ 2) $Q) \geq Q$ and the condition of Proposition 4 is satisfied.

Proof.

The map $\Psi$ can be represented as

$$
\begin{gathered}
\Psi(x)=q x+\sum_{s=1}^{l-2} \sum_{r, j:|r-j|=s, r<j}\left(q-q_{s}\right)\left(\left|e_{r}><e_{r}\right|-\left|e_{j}><e_{j}\right|\right) x \\
\cdot\left(\left|e_{r}><e_{r}\right|-\left|e_{j}><e_{j}\right|\right)+ \\
\left(q-q_{1}\right)\left(\left|e_{1}><e_{1}\right|-\left|e_{l}><e_{l}\right|\right) x\left(\left|e_{1}><e_{1}\right|-\left|e_{l}><e_{l}\right|\right) .
\end{gathered}
$$


It follows from (12) that the property $q_{j} \leq q$ garantees the complete positivity for $\Psi$.

Proof of Theorem.

The additivity conjecture for the depolarizing channel immediately follows from Proposition 3. The decreasing property of the entropy implies that

$$
S\left(\Xi^{\otimes n}(x)\right) \geq S\left(\Phi^{\otimes n}(x)\right) .
$$

Hence, to derive the result we need to prove equalities in (13). It follows from the definition (1) of the phase damping that $\Psi$ maps the projections $Q_{j}=\left|e_{j}><e_{j}\right|$ to itself, $\Psi\left(\left|e_{j}><e_{j}\right|\right)=\left|e_{j}><e_{j}\right|$. So $S\left(\Psi \circ \Phi\left(Q_{j}\right)\right)=S\left(\Phi\left(Q_{j}\right)\right)=\inf _{P \in \operatorname{Proj}(H)} S(\Phi(P))$. The result follows.

Acknowlegments. The author is grateful to Professor A.S. Holevo for a careful reading of the text and many fruitful discussions. The work is partially supported by INTAS 00-738.

\section{References}

[1] A.S. Holevo. Quantum coding theorems. Russ. Math. Surveys. 53:6 (1998) 1295-1331. LANL e-print quant-ph/9808023.

[2] G.G. Amosov, A.S. Holevo, R.F. Werner. On some additivity problems in quantum information theory. Probl. Inf. Transm. 36 (2000) 4, 24-34. LANL e-print quant-ph/0003002.

[3] G.G. Amosov, A.S. Holevo. On the multiplicativity conjecture for quantum channels. Theor. Probab. Appl. 47 (2002) no. 1, 143-146. LANL e-print math-ph/0103015.

[4] J. Cortese. The Holevo-Schumacher-Westmoreland channel capacity for a class of qudit unital channels. LANL e-print quant$\mathrm{ph} / 0211093$.

[5] A.S. Holevo. Remarks on the classical capacity of quantum channel. LANL e-print quant-ph/0212025.

[6] P. Shor. Equivalence of additivity questions in quantum information theory. LANL e-print quant-ph/0305035.

[7] A.S. Holevo, M.E. Shirokov. On Shor's channel extension and constrained channels. LANL e-print quant-ph/0306196. 
[8] C. King. Additivity for unital qubit channels. LANL e-print quant-ph/0103156. J. Math. Phys. 43 (2002) no. 10, 4641-4653.

[9] C. King. The capacity of the quantum depolarizing channel. LANL e-print quant-ph/0204172. IEEE Trans. Inform. Theory 49 (2003) no.1, 221-229.

[10] P. Shor. Additivity of the classical capacity of entanlementbreaking quantum channels. J. Math. Phys. 43 (2002) 4334-4340. LANL e-print quant-ph/0201149.

[11] A.S. Holevo, R.F. Werner. Counterexample to an additivity conjecture for output purity of quantum channel. J. Math. Phys. 43 (2002) no. 9, 4353-4357. LANL e-print quant-ph/0203003.

[12] M. Ohya, D. Petz. Quantum entropy and its use. Texts and Monographs in Physics. Springer-Verlag, 1993. 